\title{
Nasal Polyposis with Frontoethmoid Osteoma: A Rare Case Report
}

\author{
Authors \\ Pankaj Chauhan ${ }^{1}$, Disha Sharma ${ }^{2}$ \\ ${ }^{1}$ MS Otorhinolaryngology, Regional Hospital, Kullu, H.P., India \\ ${ }^{2}$ Department of Otorhinolaryngology, Dr Radhakrishnan Govt. Medical College, Hamirpur, H.P., India \\ *Corresponding Author

\section{Disha Sharma} \\ Department of Otorhinolaryngology, Dr Radhakrishnan Govt. Medical College, Hamirpur, H.P., India

\begin{abstract}
Osteoma is a rare benign lesion of the skull, with an incidence of $0.6 \%$ of all benign tumors of paranasal sinuses. It is commonly asymptomatic, and mostly diagnosed incidentally at the imaging. The most frequent symptoms are headache, nasal obstruction, nasal discharge and facial pain. Prevalence of nasal polyposis in normal population is around 1\%. It is very rare that osteoma and nasal polyposis present together. We present a case of patient with bilateral nasal polyposis with left frontoethmoid osteoma.
\end{abstract}

Keywords: Osteoma, Benign, Polyposis, Frontoethmoid.

\section{Introduction}

Osteomas are the most common fibro-osseous lesions of the head and neck region, affecting mainly the mandible, and represent the most frequent benign tumor of the paranasal sinuses ${ }^{1}$. Osteomas are generally asymptomatic, and the diagnosis is often accidental, during paranasal sinus radiological evaluation performed for other reasons. About 3\% of all paranasal sinus CT scans show osteomas, which can be easily diagnosed because of their peculiar radiological aspect ${ }^{2}$. The symptoms includes headache localized over area of involvement, nasal obstruction, rhinorrhea, anosmia, facial pain and ocular symptoms. Infective, traumatic and embryologic theories have been suggested for the development of osteoma. Associations of osteomas with colorectal polyps i.e. Gardner's syndrome ${ }^{3}$ have been described in literature but association with nasal polyposis not much described. We present a case of patient with bilateral nasal polyposis with left frontoethmoid osteoma.

\section{Case Report}

A 52 years old female patient presented with history of nasal obstruction both side associated with mucoid nasal discharge, postnasal drip, sneezing, and decreased sense of smell both side. Her past medical history revealed no co-morbidities. No history of any nasal surgery or trauma. On endoscopic nasal examination greyish nasal polyps were present both side. Computerized tomography (CT) scan of the nose and paranasal sinus revealed soft tissue density in bilateral nasal cavities, bilateral maxillary, left frontal, ethmoid and sphenoid sinuses along with hyperdense bony lesion in left frontoethmoid region suggestive of nasal polyposis along with left frontoethmoid osteoma (Fig. 1). 


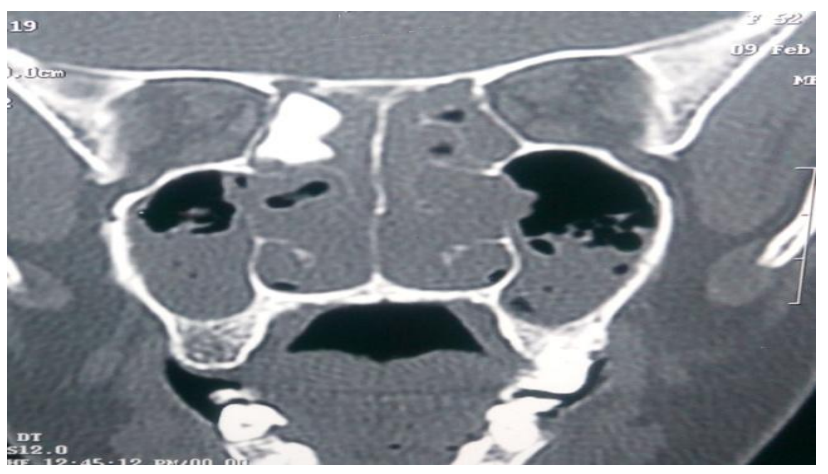

Fig. 1: Homogeneous dense osteoma (left) and soft tissue density

Frontoethmoid osteoma was successfully removed with no major operative complications using an endoscopic approach, we used intranasal drill with diamond burr. Polyps present in sinuses were removed. (Fig. 2)

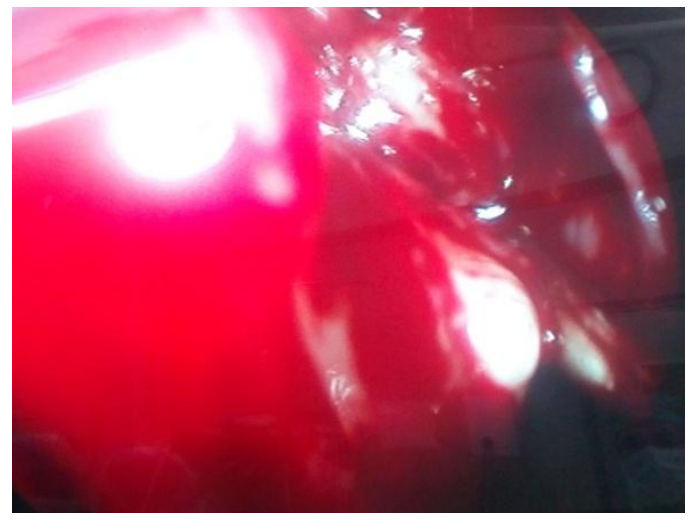

Fig. 2: Intraoperative endoscopic view showing osteoma

The cavity was packed with medicated ribbon gauze. Postoperative period was uneventful. Histopathology of the specimen was reported as osteoid osteoma with polyp. The patient was asymptomatic on follow-up.

\section{Discussion}

Osteomas are usually diagnosed incidentally during radiologic examination. The slow growth rate of osteomas explains the poor symptomatology. Only $10 \%$ of cases cause symptoms, which differ depending on the location and size of the mass ${ }^{4}$. Prevalence of nasal polyposis in normal population is around $1 \%$ however it is more commonly associated with allergy, asthma, aspirin, atopy, and cystic fibrosis and can be as high as $80 \%$ in AFRS ${ }^{5}$. Nasal polyp and osteomas very rarely occur together. There is known association between multiple osteomas and intestinal polyps, lipomas, fibromas, neurofibromas, abnormal teeth, epidermoid cyst, pigmented skin lesions (Gardner's syndrome). ${ }^{3}$ No definite etiology has been identified. The infectious theory holds that osteomas are the manifestation of bony hypertrophy resulting from a chronic state of infection due to Th2 based eosinophilic inflammation. Persistent Th-2 related inflammatory disorder of the breathing epithelium of the nasal and paranasal cavity is present in most subjects with either allergic or nonallergic chronic hyperplastic rhinosinusitis. These patients show an unusual association between nasal polyposis and paranasal osteoma. ${ }^{6}$ Osseous metaplasia in mucosal polyps has been reported in the external auditory canal, tongue, stomach and intestines but in nasal polyps it is rare phenomenon. Bone morphogenetic proteins and transforming growth factor beta-1 have been shown to be involved in ectopic bone formation, and therefore may be involved in ossification of mucous polyps. Squamous metaplasia of respiratory epithelium covering nasal polyp is common. De vries postulated the new bone formation occurring in nasal polyps arose from bony remnants left behind during previous surgery. ${ }^{7}$ CT scan examination allows the differential diagnosis between fibro-osseous lesions of the skull and paranasal osteoma. These lesions, which include fibrous osteoma, ossifying fibroma and fibrous dysplasia may have a similar radiographic appearance, but their borders are less well-defined than those of osteoid osteoma. ${ }^{8}$ The surgical treatment of ethmoid osteomas remains controversial. It is taken into consideration when symptoms are present or when a rapid growth leads to potential obstruction of the nasal-frontal duct, orbital and skull invasion. ${ }^{9}$ In our patient the polyp shows no histologically abnormal epithelium overlying the bone formation and exact etiology of osteoma along with nasal polyposis is still matter of debate. 


\section{Conclusion}

This is very presentation of bilateral nasal polyposis and left sided frontoethmoid osteoma. After going through the literature, bone changes start primarily in the ethmoid sinus and associated with nasal polyps. Their occasional resemblance to malignancy also empha size the importance of histologically assessing all polypoid material post-operatively. In conclusion, we recommend further studies to confirm whether bony changes related to nasal polyposis do increase the risk of developing osteomas of the skull.

\section{References}

1. Karatas A, Cebi IT, Yanik T, Kocak A, Selcuk T. Osteoma Originating from Mastoid Cortex. Turk Otolarengoloji Arsivi/ Turkish Arch Otolaryngol 2017;55(1):48-50.

2. Janovic A, Antic S, Rakocevic Z, Djuric M. Paranasal sinus osteoma: is there any association with anatomical variations? Rhinology 2013;51(1):54-60.

3. Gardner EJ, Plenk HP. Hereditery pattern for multiple osteomas in a group. Amer J Hum Gen 1952;4:31-35.

4. Erdogan N, Demir U, Songu M, Ozenler NK, Uluç E, Dirim B. A prospective study of paranasal sinus osteomas in 1,889 cases: changing patterns of localization. Laryngoscope 2009;119(12):2355-59.

5. Drake lee AB, Lowe D, Swanstone A. Clinical profile and recurrence of nasal polyps. J Laryngol Otol 1984;98:783-93.

6. Bachert C, Zhang L, Gevaert P. Current and future treatment options for adult chronic rhinosinusitis: Focus on nasal polyposis. J Allergy Clin Immunol 2015;136(6): 14311440.

7. De vries. New bone formation in nasal polyps. Rhinology 1988;2:21,109-12.

8. Sontakke SA, Karjodkar FR, Umarji HR. Computed tomographic features of fibrous dysplasia of maxillofacial region. Imaging Sci Dent 2011;41(1):23-28.
9. Chahed H, Hachicha H, Bachraoui R. Paranasal sinus osteomas: Diagnosis and treatment. Rev Stomatol Chir Maxillofac Chir Orale 2016;117(5): 306-310. 